\title{
Rates of hospital admissions for primary angle closure glaucoma among Chinese, Malays, and Indians in Singapore
}

\author{
Tien Yin Wong, Paul J Foster, Steve K L Seah, Paul T K Chew
}

Singapore National Eye Center, Singapore T Y Wong

P J Foster

S K L Seah

P T K Chew

Department of

Ophthalmology,

National University of

Singapore

T Y Wong

P T K Chew

Department of Ophthalmology and Visual Sciences,

University of

Wisconsin-Madison,

Wisconsin, USA

T Y Wong

Department of Epidemiology, Johns Hopkins University,

Baltimore, Maryland, USA

T Y Wong

Institute of

Ophthalmology,

University College

London

P J Foster

Correspondence to: Tien Yin Wong, Department of Ophthalmology, National

University of Singapore, 10

Kent Ridge Crescent,

Singapore 119260

tienyinwong@yahoo.com

Accepted for publication 13 April 2000

\begin{abstract}
Aim-To estimate the rates of hospital admissions for primary angle closure glaucoma (PACG) in Chinese, Malays, and Indians in Singapore

Methods-A population-wide hospital discharge database in Singapore was used to identify all hospital admissions with a primary discharge diagnosis of PACG (International Classification of Disease-CM code: 365.2 ). The Singapore census was used for denominator data.

Results-Between 1993 and 1997 there were 894 hospital admissions for PACG. The mean annual rate of PACG admissions was 11.1 per 100000 (95\% confidence interval (CI), 10.4, 11.8) among people aged 30 years and over. The annual rate was highest for Chinese (age and sex adjusted rate: 12.2 per 100000 ), which was twice that of Malays (6.0 per 100000$)$ and Indians (6.3 per 100 000). Females had two times higher rates than males in all three races (age adjusted relative risk: $2.0,95 \%$ CI: 1.7, 2.3).

Conclusion-Malay and Indian people had identical rates of hospital admissions for PACG, which were only half the rates compared with Chinese.

(Br F Ophthalmol 2000;84:990-992)

Primary angle closure glaucoma (PACG) is an important cause of blindness in east Asian people. ${ }^{1-3}$ A previous study in Singapore found an incidence of symptomatic primary angle closure (PAC) of 12.2 per 100000 per year, with Chinese being at three times greater relative risk compared with non-Chinese (Malay, Indian, and other ethnic groups). ${ }^{4}$ The small number of non-Chinese people $(\mathrm{n}=16)$ prevented us from obtaining a reliable estimate of the incidence in Malay and Indian people. This information may be important as more than one fifth of the world's population is of either South East Asian origin (including Indonesian, Thai, Vietnamese, and Malay people) or from the Indian subcontinent. ${ }^{5}$ The purpose of this report is to estimate the rates of PACG in the Malay and Indian population in Singapore using hospitalisation data.
\end{abstract}

\section{Material and methods}

Singapore has a stable multiethnic resident population of 3.16 million people, of whom $77 \%$ are Chinese, $14 \%$ Malay, and $8 \%$ Indians, and $1 \%$ other ethnic groups. The study population consisted of citizens and permanent resi- dents of Singapore. We used a national hospital discharge database, linked to a national medical savings (Medisave) database to identify cases of PACG admitted to hospital. The hospital discharge and Medisave database have been described in previous reports. ${ }^{67}$ Briefly, all patients admitted to Singapore hospitals are assigned specific discharge diagnoses coded using the International Classification of Diseases, Ninth Revision-Clinical Modification codes (ICD-9-CM codes). In this study, the definition of a case of PACG was any patient requiring hospital admission with a principal discharge diagnosis of PACG (ICD-9-CM diagnoses code of “365.2”). Quality control measures, including annual audits in all hospitals, are routinely taken by the Professional Medisave Audit Unit in the Ministry of Health to ensure accuracy of the data. ${ }^{6} 7$

For the denominator, population data were drawn directly from the 1990 Singapore census and 1995 mid-decade census (Department of Statistics, Ministry of Trade and Industry, Singapore). Figures for all other years in our study (1993, 1994, 1996, and 1997) were based on projections by the Department of Statistics. Ethnic groups were defined in the census according to one of four main ethnic groups: Chinese (refers to people of Chinese origin such as Hokkien, Teochew, Cantonese, Hakka, etc), Malays (refers to people of Malay or Indonesian origin such as Javanese), Indians (refers to people with ancestry originating on the Indian subcontinent), and others (comprising all people other than the first three categories such as European, Eurasian, Middle Eastern, and Japanese). People of mixed heritage were classified under the ethnic group of their fathers. As the "others" category includes multiple ethnic populations and is not entirely compatible between the hospital discharge database and the census; incidence data on this group were not analysed.

Average annual rates of PACG admissions for each subgroup (10 year age, sex, and ethnic groups) were calculated by dividing the number of admissions with the 5 year average population of that subgroup. Age and sex adjusted rates were calculated by direct adjustment using the total population in 1995. Relative risks and $95 \%$ confidence intervals ( $95 \%$ CI) were calculated based on the Poisson distribution. The institutional review board of the Singapore National Eye Centre approved this study. 


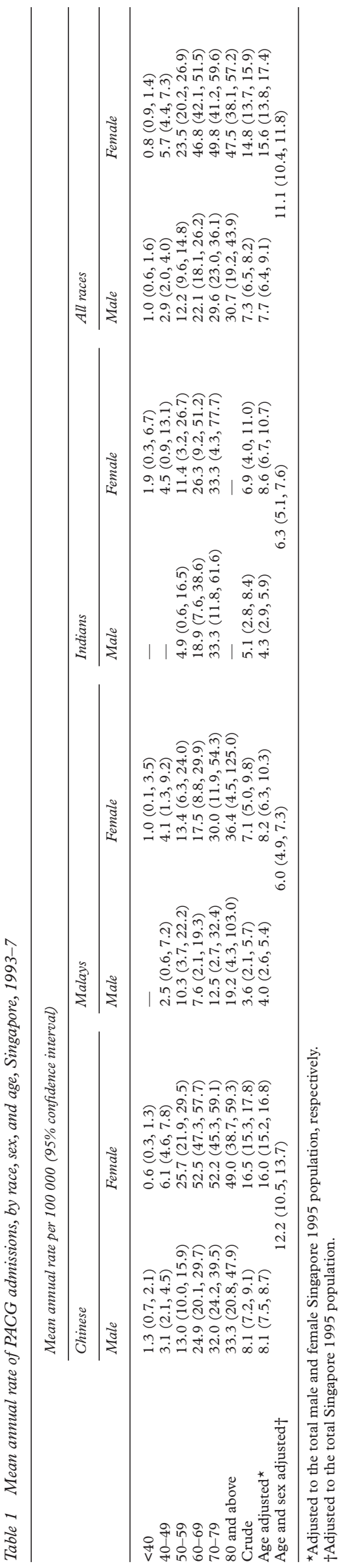

\section{Results}

Between 1 January 1993 and 31 December 1997, there were 894 hospital admissions for PACG. The numbers for each individual year were: 185 (1993), 167 (1994), 193 (1995), 183 (1996), and 166 (1997). Excluded from further analyses were 28 residents classified in the "others" ethnic category. Of the remaining 866 admissions, 785 (90.6\%) were Chinese, 51 (5.9\%) were Malays, and 30 (3.5\%) were Indians. More than two thirds were females $(n=583)$.

The mean rate of hospital admissions attributable to PACG was 11.10 per 100 000/year (95\% CI: 10.4, 11.8) in people aged 30 years and older. The variation over the 5 years was low (range 10.1 per 100000 in 1997 to 12.1 per 100000 in 1996) and no distinct time trends were observed (linear regression coefficients of rates by time: $\alpha=11.6, \beta=-0.18$ (95\% CI: $-1.08,0.72)$ )

There were marked variations in the rate of PACG admission by age, sex, and ethnic group (Table 1). The rate was highest for Chinese people, with a mean age and sex standardised incidence of 12.2 per 100 000/year in those aged 30 years and older. The rates in Malay (6.0 per 100000$)$ and Indian people (6.3 per 100 000) were similar. Women had an age adjusted rate of hospital admissions twice that of men, with the higher rates seen in all three ethnic groups. There was an age dependent increase in rates that was similar across race and sex.

\section{Discussion}

Our study provides new information on the morbidity from PACG in Malays and Indians, two groups of Asian people for whom epidemiological data on glaucoma are lacking. ${ }^{1}$ As our study was based on hospital admissions, our estimates reflect more accurately the incidence of symptomatic PAC in the population, rather than the actual incidence of PACG. Nevertheless, we found that the rate of symptomatic PAC among Malays and Indians to be nearly identical, and only half that of Chinese. The rate in Malay people (6.0 per $100000 /$ year) is similar to that of symptomatic PAC in another ethnic South East Asian population in Thailand (7.0 per 100 000/year). ${ }^{8}$ In addition, we confirmed once more the strong relation between PACG, increasing age, and female sex in all three ethnic groups. ${ }^{4}$

The results of this present study should be contrasted with our previous study on symptomatic PAC. ${ }^{4}$ The strengths of this current study included a nationwide identification of cases over a 5 year period, completeness of coverage of all hospital admissions, and accurate records. ${ }^{67}$ However, there were some important limitations. Firstly, cases of PACG not admitted to hospitals were not captured. Secondly, the cases identified will contain a mixture of symptomatic PAC (people presenting with an acute, symptomatic exacerbation of PAC) and those with PACG who are undergoing elective surgery. As the latter group is likely to be small (most elective ophthalmic surgeries are day cases), we believe our data will give a 
good estimate of the rate of symptomatic PAC. Our previous study, on the other hand, was based on a prospective registry collecting symptomatic PAC cases reported by Singapore ophthalmologists over a 1 year period. ${ }^{4}$ While symptomatic PAC cases not requiring hospitalisation were captured, limitations of that study included a reliance on voluntary participation by all ophthalmologists, and the inability to calculate precise Malay and Indian specific rates owing to the small number of symptomatic PAC cases in non-Chinese. In the current study, the mean annual number of hospital admissions for symptomatic PAC was 179 , while in the previous study, the annual number of reported symptomatic PAC was 189. Therefore, the current study underestimates the rate of symptomatic PAC in the previous study by approximately 10 cases per year $(5.2 \%)$.

Inferences on the racial variation observed must be made in context with the assumption that access to hospitalisation was approximately equal among the three races in Singapore. This assumption appears reasonable given that hospitalisation costs can be paid by Medisave in $90 \%$ of the population, with subsidised hospital care available to the remaining $10 \%$ who do not have a Medisave account. ${ }^{9}$ For conditions requiring acute hospital care, such as symptomatic PAC, racial variation in access to health care is even less likely. As a comparison, using the same database over the same period (1993-7), the average rates of hospital admissions for acute appendicitis (ICD-CM-9 code: 540.0) for Chinese, Indians, and Malays were: $61.6,66.8$, and 57.8 per
100 000/year, respectively (unpublished data, Ministry of Health, Singapore, 1998).

Although the number of cases described here seems relatively small when compared with the number of glaucoma patients worldwide, ${ }^{1}$ the public health impact is potentially enormous. PACG probably has a far higher propensity to cause blindness than open angle glaucoma and cases of symptomatic PAC usually require urgent medical attention and hospitalisation. By cautiously extrapolating our data to other countries in Asia, we could expect 150000 hospital admissions per year in China, 55000 in India, and 12000 in Indonesia for symptomatic PAC alone. Clearly, there is a need to design and implement appropriate eyecare strategies to manage this condition in Asia.

1 Quigley HA. Number of people with glaucoma worldwide. Br f Ophthalmol 1996;80:389-93.

2 Baasanhu J, Johnson GJ, Burendei G, et al. Prevalence and causes of blindness and visual impairment in Mongolia: a survey of populations aged 40 years and older. Bull World Health Organ 1994;72:771-6.

3 Foster PJ, Baasanhu J, Alsbirk PH, et al. Glaucoma in Mongolia-a population-based survey in Hövsgöl Province, Northern Mongolia. Arch Ophthalmol 1996;114:

4 Seah SKL, Foster PJ, Chew PT, et al. Incidence of acute primary angle-closure glaucoma in Singapore. An island-wide survey. Arch Ophthalmol 1997;115:1436-40.

5 The World Bank. World development report 1997: the state in a changing world. New York: Oxford University Press, 1997:214-15.

6 Wong TY, Tielsch JM, Schein OD. Racial difference in the ncidence of retinal detachment in Singapore. Arch Ophthalmol 1999;117:379-83.

7 Wong TY, Tielsch JM. A population-based study on the incidence of ocular trauma in Singapore. Am f Ophthalmol 1999;128:345-51.

8 Fujita K, Negishi K, Fujiki K, et al. Epidemiology of acute angle-closure glaucoma: report 1. Fpn $\mathcal{F}$ Clin Ophthalmol 1996;37:625-9.

9 Phua KH. Savings for health. World Health Forum 1987;8: 39-41. 